\title{
Limit Cycle Oscillation Analysis on the Design of Wind Power Harvester with Fluttering Aerofoil
}

\author{
C. P. Pagwiwoko ${ }^{1}$ \\ ${ }^{1}$ Department of Mechanical, Materials and Manufacturing Engineering \\ Faculty of Engineering \\ University of Nottingham Malaysia Campus \\ Jalan Broga - Semenyih 43500, Selangor Darul Ehsan (Malaysia) \\ Phone/Fax number:+603 8924 8166/8017, e-mail: Cosmas.Pagwiwoko@ nottingham.edu.my
}

\begin{abstract}
This paper presents a new concept of wind power harvester which extracts the energy from the flow through an aeroelastic vibration phenomenon. Different from traditional rotating wind turbines, a fluttering aerofoil in the form of transversal and rotational motions is used in the process. Analysis of flutter characteristics in frequency domain is carried out to determine the flutter stability boundary. The energy is extracted at the wind speed above the flutter boundary where the system may become nonlinear. The operating range of the device is set up at a stabilized oscillation in the nonlinear zone by regulating the pitching motion limited to a certain angular travelling distance.
\end{abstract}

\section{Key words}

wind energy, aeroelasticity, flutter boundary, self-excited vibration, limit cycle oscillation, bond graph.

\section{Introduction}

Wind energy is advantageous because it is green since no carbon foot print and cheaper than solar energy per unit power. It was reported recently that $2 \%$ electrical energy produced by wind powered generators worldwide and the capacity has doubled within last 3 years. Rotating wind turbine is the most common device to harvest the wind energy. Although it is quite attractive the wind energy generators become less efficient when scaled down because the normal rotating turbines require bulky gearing system that introduces mechanical frictions. This type of device performs well when the wind is strong, however at low wind speed the performance drops drastically. This is because conventional electromagnetic wind turbines require rotating fins and gearing which adds bulk, and they become less efficient when scaled down [1].

Recently, the design of non-rotating turbine known as wind-belt, based on the principle of aeroelastic flutter showed a remarkable result in harvesting the energy from air flows [6]. In this concept an aeroelastic vibration occurs on a thin flexible structure when elastic and inertial forces of the structure interact with the excitation force induced by aerodynamic flow. This type of vibration can be unstable divergent, where above a critical speed of flow the energy encountering the flexible structure manifested as dynamic response cannot be dissipated any longer and it may lead to the destruction of the structure. Whilst this kind of oscillation is usually considered unsafe and needs to be avoided in the design of light weight structures such as aircraft wing and suspension bridge, some engineers invented the energy harvester based on flutter effect, especially to improve the low wind speed efficiency.

\section{Design Concept of the Harvester}

Aerodynamic forces acting on a moving lifting surface are divided into two parties i.e. the component that supports the structural movement of the body and the other that resists the movement. When the aerodynamic and structural loads are in balance, it will produce a harmonic oscillation. This kind of vibration happens at certain speed of flow so-called the flutter boundary. Above this critical flow speed, there is an unbalance of energy flowing to the structure that cannot be dissipated, where in consequence the oscillation grows divergently until disintegration of the structure. This self-excitation phenomenon is utilized in the concept of the harvester such of wind-belt, to extract the energy from the flow. The extracted energy is then converted into electrical power through a power transducer installed on the moving part of the device.

The idea of our research is to ameliorate the performance of the harvester by streamlining the shape of the oscillating surface in order to reduce the aerodynamic drag due to flow separation that occurs in the wind-belt device. This novel device is designed as a rigid but slender beam with an aerofoil cross-section sitting on a flexible support. This suspension allows the panel to move in heaving and pitching aligned with the oncoming the flow. This aeroelastic model is explained schematically in Fig. 1.

The two mode frequencies of the motions move close to each other as the wind speed increases until the internal resonance takes place. The classical flutter appears when the two modes coalesce where there is an exchange of energy between the two modes. The damping factors of the modes also vary with the flow speed. When the wind speed exceeds the critical speed of flutter, the value of the 
aerodynamic damping in the analysis becomes negative, interpreted as the energy flows into the structure.

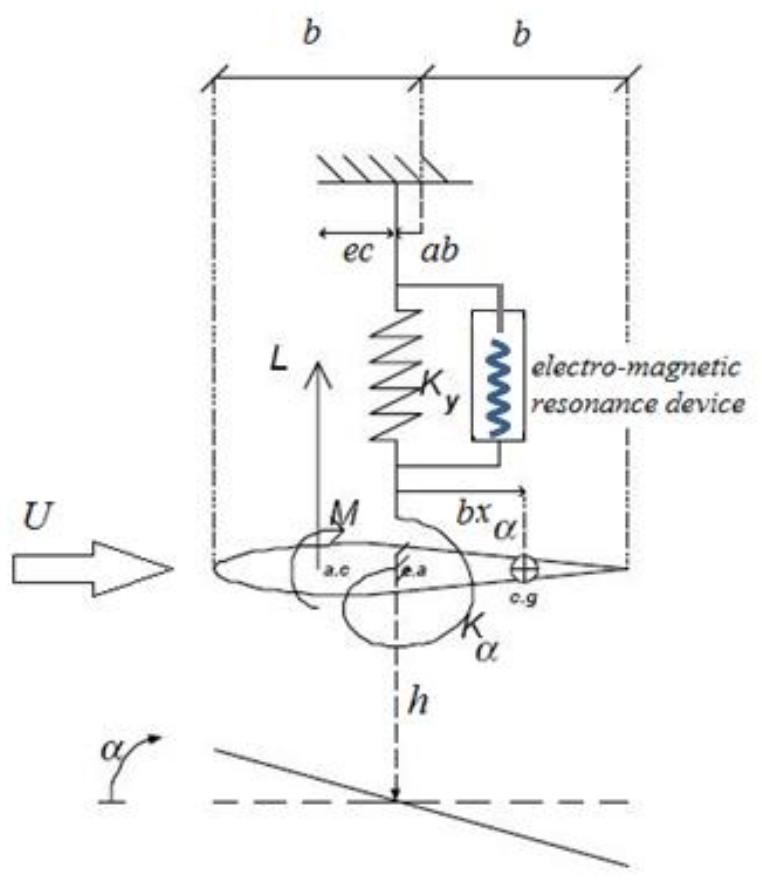

Fig. 1. Schematic showing of the aerofoil with heave and pitch spring restraints. Electric power generator is installed to the system to convert the vibration energy in heaving mode.

Prototype 1 shown in Fig. 2 was fabricated and then tested in wind-tunnel to evaluate the effectiveness of the concept. The modes of the motion in transversal and rotational are enabled by flexible support mechanism located at the both ends. This design is considered more effective compared with wind-belt device since only certain selected vibration modes in heaving and pitching to be tuned to generate a classical flutter at a particular wind speed.

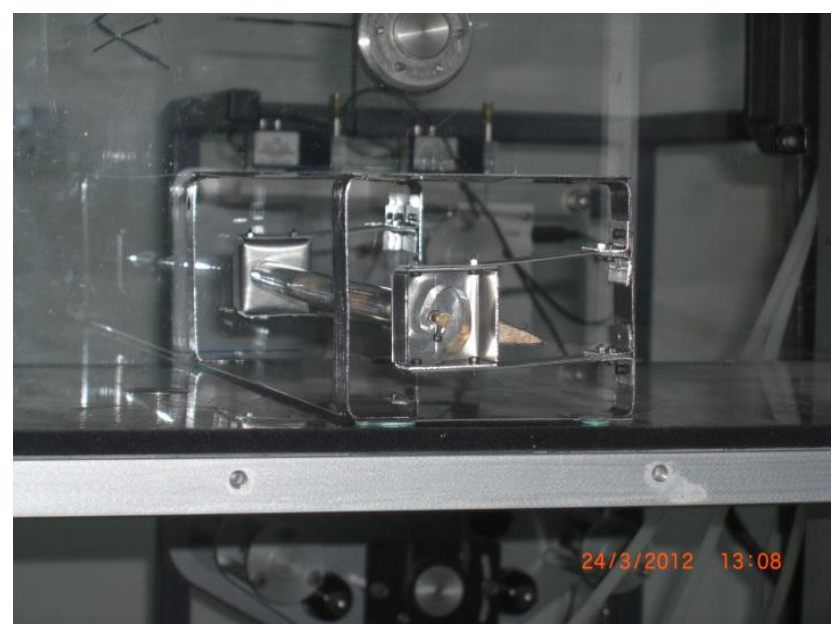

Fig. 2. Wind-tunnel flutter model of Prototype 1 with heaving and pitching modes. Two pairs of cantilevers installed at both sides of the lifting surface enable the aerofoil to move in heaving and the worm springs fixed in the casing to the shaft allow the motion in pitching.

A second wind-tunnel model made of solid aluminum was then fabricated with slight different design of suppot system but still ensuring the same motions in the twodegree-of-freedom. With a bigger magnitude in mass and inertia the device is expected to extract more energy from the flow at the same flow speed.

In this second design, the rotational motion is restrained by a pair of pre-tensioned strings at each side rather than a worm spring. The configurations of the flexible supports determine the dynamic parameters of the model: spring constance in heaving and pitching, elastic axis position and the inertial coupling of the mass. The parameters of this flutter model is as shown in Table 1.

Table 1. - Model Parameters

\begin{tabular}{|l|c|c|}
\hline \multicolumn{1}{|c|}{ Parameter } & Notation & Value \\
\hline Aerofoil mass & $m$ & $0.932 \mathrm{~kg}$ \\
Mass moment of inertia & $I_{\alpha}$ & $7.8545 \mathrm{e}-4 \mathrm{~kg} \mathrm{~m}{ }^{2}$ \\
Inertial coupling & $m x_{\alpha} b$ & $0.0159 \mathrm{~kg} \mathrm{~m}$ \\
Aerofoil mass offset & $a b$ & $-0.0125 \mathrm{~m}$ \\
Chord length & $c$ & $0.1 \mathrm{~m}$ \\
Span length & $l$ & $0.28 \mathrm{~m}$ \\
Transversal stiffness & $k_{h}$ & $512 \mathrm{~N} / \mathrm{m}$ \\
Rotational stiffness & $k_{\alpha}$ & $1.7254 \mathrm{Nm} / \mathrm{rad}$ \\
Location of elastic axis & $a$ & -0.25 \\
Location of c.g & $x_{\alpha}$ & 0.341 \\
Aerofoil type & NACA 0018 & \\
Coefficient of lift & $d / d \alpha C_{L}$ & $2 \pi(\mathrm{small} \alpha)$ \\
pitching moment at $1 / 4 c$ & $d / d \alpha C_{m}$ & $0(\mathrm{small} \alpha)$ \\
Air density & $\rho$ & $1.225 \mathrm{~kg} / \mathrm{m}^{3}$ \\
\hline
\end{tabular}

\section{Flutter Analysis}

The equation of motion of the aero-elastic model shown in Fig. 1 having two-degree-of-freedom motions in heaving and pitching indicated by the coordinates $h$ and $\alpha$ is presented as Equation (1) below:

$$
\begin{aligned}
{\left[\begin{array}{cc}
m & m x_{\alpha} b \\
m x_{\alpha} b & I_{g}+m\left(x_{\alpha} b\right)^{2}
\end{array}\right]\left\{\begin{array}{l}
\ddot{h} \\
\ddot{\alpha}
\end{array}\right\}+\left[\begin{array}{cc}
k_{h} & 0 \\
0 & k_{\alpha}
\end{array}\right]\left\{\begin{array}{l}
h \\
\alpha
\end{array}\right\} } & =\left\{\begin{array}{c}
-L \\
L e c+M_{a c}
\end{array}\right\} \\
& =\left\{F_{\text {aero }}\right\}
\end{aligned}
$$

The aerodynamic loads consist of lift $L$ and pitching moment $M$ are considered work on the aerodynamic centre of the aerofoil ( $1 / 4$ of chord from the leading edge), $b$ is $1 / 2$ chord. These loads depend proportionally to the dynamic pressure $1 / 2 \rho U^{2}$ and to the angle of attack. For a moving lifting surface, the vertical velocity relative to the oncoming velocity has to be taken into account in defining the angle of attack, and for the case of harmonic motion Theodorsen function $C(k)$ is to be applied to the expressions of the loads [4].

The lift and pitching moment are as shown in equations (2) and (3).

$$
\begin{gathered}
L=\frac{1}{2} \rho U^{2} c l C(k) \frac{\partial C_{l}}{\partial \alpha}\left(\alpha+\frac{\dot{h}}{U}\right) \\
M_{a c}=\frac{1}{2} \rho U^{2} c^{2} l C(k) \frac{\partial C_{m}}{\partial \alpha}\left(\alpha+\frac{\dot{h}}{U}\right)
\end{gathered}
$$


In harmonic motions of an aerofoil there is a lag between the motion of the body and the force generated by the pressure around the section. Theodorsen's function $C$ is used to model the changes in amplitude and phase of the sinusoidal unsteady aerodynamic forces relative to the quasi-steady forces for different reduced frequencies. The parameter of reduced frequency is defined as $k=\omega b / U$. The function $C$ constructed from Hankel and modified Bessel functions in complex form $(F+i G)$ as a function of $k$ is presented in Fig. 3 below.

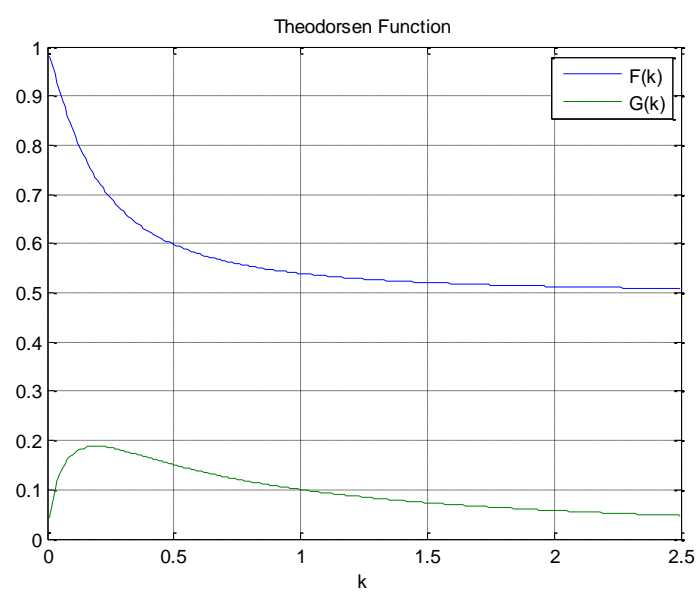

Fig. 3. Theodorsen's function $C(k)=F(k)+i G(k)$

In case of the harmonic oscillating motion of an aerofoil, the right-hand-side of equation (1) the aerodynamic loads $F_{\text {aero }}$ can be expressed in term of reduced frequency $k$ and the amplitudes of the motion in heaving and pitching, as expressed in equation (4) below.

$$
\left\{F_{\text {aero }}\right\}=\left\{\begin{array}{c}
-L \\
L e c+M_{a c}
\end{array}\right\}=\frac{1}{2} \rho U^{2}[Q(i k)]\left\{\begin{array}{l}
h \\
\alpha
\end{array}\right\}
$$

Considering the expressions of the lift in equation (2) and the pitching moment acting at the aerodynamic pressure of the aerofoil in equation (1), the matrix of aerodynamic forces $Q$ in term of reduced frequency $k$ can be stated in a complex form as:

$$
[Q(i k)]=c l C(k) \times
$$

$$
\left(\left[\begin{array}{cc}
0 & \left(-\frac{\partial C_{l}}{\partial \alpha}\right) \\
0 & \left(2 \frac{\partial C_{l}}{\partial \alpha} b e+2 \frac{\partial C_{m}}{\partial \alpha} b\right)
\end{array}\right]+i\left[\begin{array}{cc}
\left(-\frac{\partial C_{l}}{\partial \alpha} \frac{k}{b}\right) & 0 \\
\left(2 e k \frac{\partial C_{l}}{\partial \alpha}+2 k \frac{\partial C_{m}}{\partial \alpha}\right) & 0
\end{array}\right]\right)
$$

In flutter analysis using " $k$ Method", it is proposed to introduce a virtual structural damping variable $g$ in the equation of motion as described in Equation (6) so the problem can be analysed in frequency domain with constant amplitude of harmonic oscillation.

$$
\begin{aligned}
{\left[\begin{array}{cc}
m & m x_{\alpha} b \\
m x_{\alpha} b & I_{g}+m\left(x_{\alpha} b\right)^{2}
\end{array}\right]\left\{\begin{array}{l}
\ddot{h} \\
\ddot{\alpha}
\end{array}\right\}+(1+i g)\left[\begin{array}{cc}
k_{h} & 0 \\
0 & k_{\alpha}
\end{array}\right]\left\{\begin{array}{l}
h \\
\alpha
\end{array}\right\} } \\
\left.\left.=\frac{1}{2} \rho U^{2}[Q(i k)]\right\} \begin{array}{l}
h \\
\alpha
\end{array}\right\}
\end{aligned}
$$

The variable $g$ has a correlation with the amount of energy to be fed in or taken out from the system such to maintain the oscillating motion. Thus the solution of the problem that gives the zero value of $g$ or a harmonic response without any power supply from outside of the system, will show the flutter unstable boundary.

Applying a harmonic expression of the response for the solution of the equation of motion expressed in Equation (6) with the oscillation frequency of $\omega$ will lead to an eigen-value problem as shown in the Equation (7) below:

$$
\begin{array}{r}
{\left[\begin{array}{cc}
k_{h} & 0 \\
0 & k_{\alpha}
\end{array}\right]^{-1}\left(\left[\begin{array}{cc}
m & m x_{\alpha} b \\
m x_{\alpha} b & I_{g}+m\left(x_{\alpha} b\right)^{2}
\end{array}\right]+\frac{\rho b^{2}}{2 k^{2}}[Q(i k)]\right)\left\{\begin{array}{l}
\hat{h} \\
\hat{\alpha}
\end{array}\right\}} \\
=\frac{(1+i g)}{\omega^{2}}\left\{\begin{array}{c}
\hat{h} \\
\hat{\alpha}
\end{array}\right\}
\end{array}
$$

It can be seen from the equation above that the eigenvalue of the system are complex. The real part of each eigen-value corresponds with the frequency of the motion and the imaginary part relates to the damping factor. In the study case of the flutter model which parameters shown in Table 1, the frequencies of the both modes as a function of wind speed can be determined by interpolating a certain range of reduced frequency $k$.

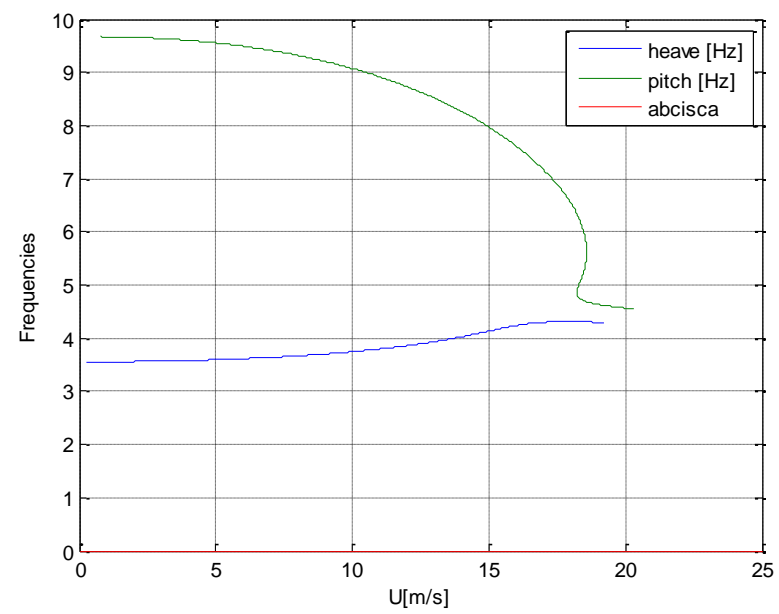

Fig. 4. Frequencies of the aerofoil in heaving and pitching modes versus wind speed.

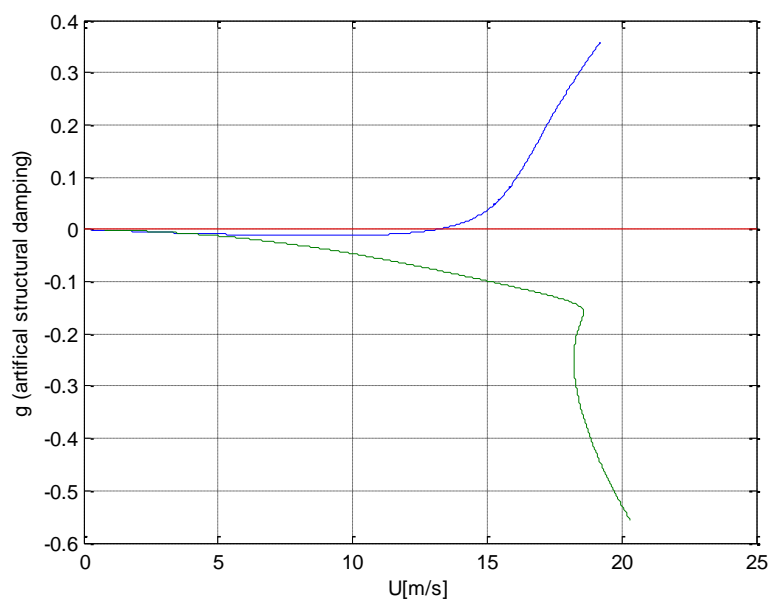

Fig. 5. Artificial structural damping factors in heaving and pitching modes versus wind speed. 
The similar procedure is conducted to obtain the artificial structural damping by solving Equation (7).

Fig. 4 shows the frequencies of the both modes of vibration as a function of wind speed while Fig. 5 shows the related artificial structural damping.

The critical flutter speed can be determined in Fig. 5 where artificial damping tends to zero. Below this speed, the negative values of the damping signify the certain amount of energy to be supplied to the system in order to generate a harmonic oscillation response and above that speed to be dissipated to maintain a stable response. It is also interesting to see the variation of both frequencies as the wind speed getting close to the flutter boundary. In Figure 4 as the aerofoil starts fluttering the two curves of frequency move close to each other explaining that an internal resonance occurs with exchange of energy between the two modes.

\section{Unsteady Aerodynamic Model}

In order to be able to analyse flutter response in time domain, a model of unsteady aerodynamic force in Laplace variable is needed to represent the aerodynamic load for an arbitrary motion of the aerofoil.

In this work a model of fraction rational function based on Roger's approximation is utilized for the purpose [4] as shown in equation (8) below:

$$
[Q(i k)] \approx\left[A_{0}\right]+\left[A_{1}\right]\left(\frac{b s}{U}\right)+\left[A_{2}\right]\left(\frac{b s}{U}\right)^{2}+\sum_{j=1}^{n} \frac{\left[A_{j+2}\right] s}{s+\beta_{j} \frac{U}{b}}
$$

The matrix coefficient of $A_{0}, A_{1}$ and $A_{2}$ have physical signification as an additional stiffness, damping and inertia due to the aerodynamic flow respectively, while $A_{j+2}$ contribute as lagging terms. The lagging coefficients $\beta_{j}$ are ratios which values and numbers are extensively chosen to ameliorate the approximation. In this work 4 coefficients are selected as $\beta_{1}=0.1, \quad \beta_{2}=0.2, \quad \beta_{3}=0.3$, $\beta_{4}=0.4$. The coefficients of the aerodynamic matrix are determined by applying the technique of least square to fit the approximation function in $s$ variable to the calculated aerodynamic matrix in frequency-domain for a certain range of $k$ in the complex Laplace plane.

The representation of the aerodynamic forces in Laplace variable $s$ as a transfer function enables to express the external loads due to the input of lifting surface motion and therefore the dynamic response of structure as a result in time domain. Fig. 6 shows results of the curve fitting technique and the accuracy of the approximation function in Laplace variable in representing the matrix of aerodynamic forces for the two motions of heaving and pitching. The transformation to the time domain is accomplished by transforming from the Fourier form into Laplace domain. One may notice that the matrix coefficient of $A_{0}, A_{1}$ and $A_{2}$ ensued from this approximation capture the dependence of the unsteady aerodynamic loads on displacement, velocity and acceleration.

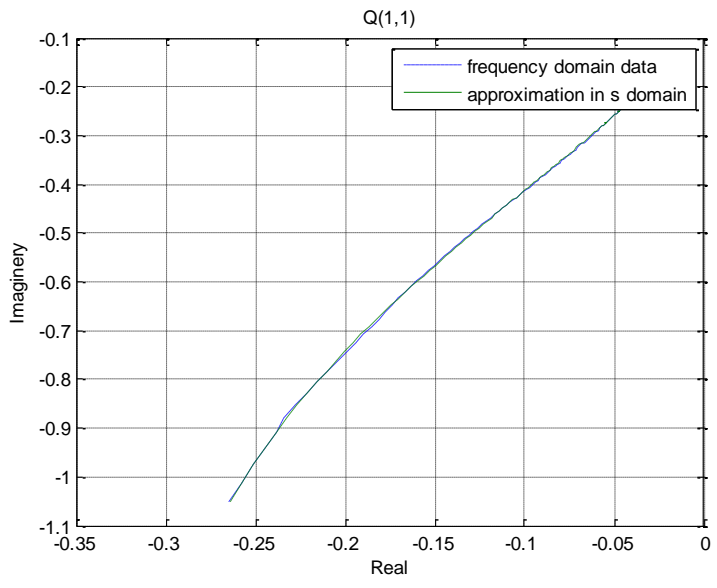

Fig. 6. Roger's approximations for the aerodynamic load coefficient matrix $Q_{11}$

The representation of the aerodynamic model as a block diagram in Simulink-Matlab can be represented in Fig.7. The input variables of the system are the transversal and rotational velocities of the aerofoil and the outputs are the lift and pitching moment. Coupled with another subsystem of dynamic motion of the aerofoil the whole system presents an interaction of flow-structure that can be observed in time domain as shown in Fig. 8.

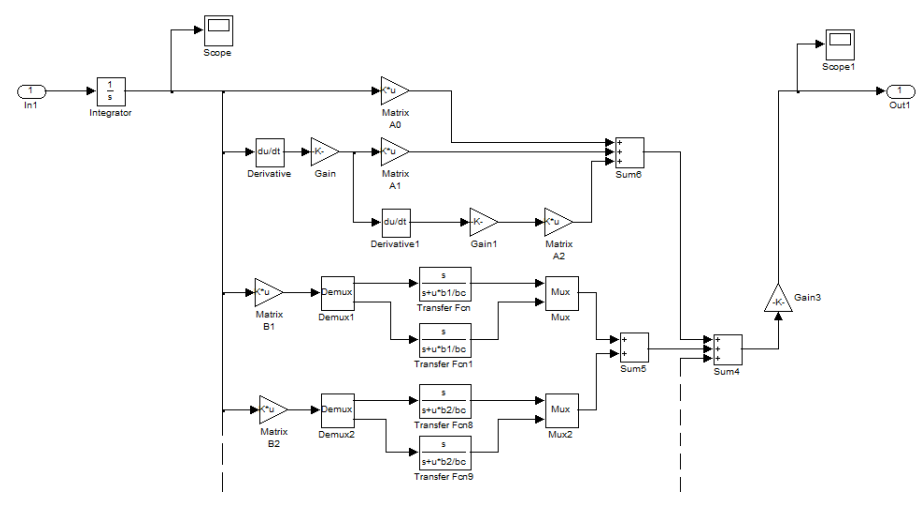

Fig. 7. Implementation of unsteady-aerodynamic load in Simulink-Matlab. Note: the matrix $B_{1}, B_{2} \ldots$ in the block diagram are matrix $A_{3}, A_{4}, \ldots A_{n}$ in equation (8).

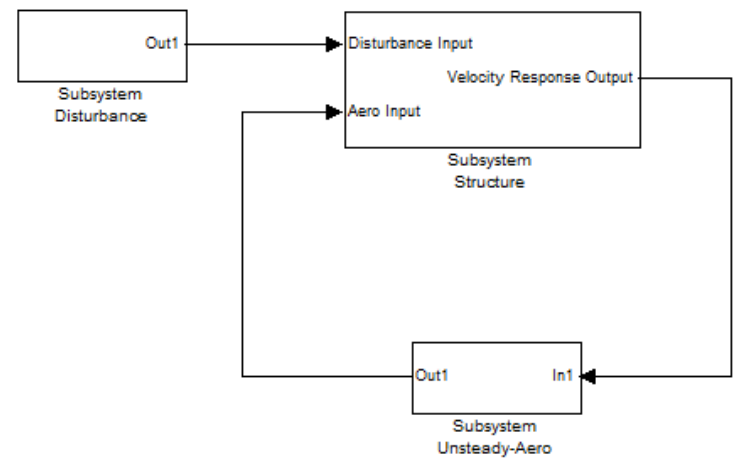

Figure 8. Implementation of Flow-Structure Interaction in Simulink-Matlab. A small disturbance in the form of impulse is needed to trigger the self-excited divergent vibration. 


\section{Dynamic Model of Flow-Structure Interaction}

The sub-system of structure coupled with the unsteadyaerodynamics excitation shown in Fig. 8 describes a fluidstructure interaction with flow-induced vibration as a result. A small disturbance is needed to initiate the simulation in evolving time especially to examine the stability. The nature of disturbance can be a small impulse imposed to the panel or a slight turbulence of flow.

The transient response of the aerofoil due the disturbance will subsequently create the aerodynamic pressure around the profile. The unsteady-aerodynamic subsystem shown in Figure 8 calculates the lift and pitching moment. This transient loads consecutively excite the aerofoil that mounted on the flexible support system and as a result produce the dynamic response and generate the new aerodynamic loads that turn the process into a closed loop. In this research bond graph modelling is introduced to analyse the system as an integrated/unified form. [3]

The dynamic modelling technique considers that a dynamic system consists of interacting sub-systems and components where all the elements within the system are connected to each other by energy bonding through which the power flows. The types of the basic elements that constructing the system are: the storing and dissipating energy components, energy transmitters (transformer/ gyrator), and junctions (serial and parallel types). A source of energy is applied to the system as the external excitations. For the case of aeroelastic problems where the external excitation is unsteady-aerodynamic loads which is the function of the structural response, the energy source component has the feedback input signals from the model, therefore it can be considered as a filter or transfer function block as explained in Fig. 8.

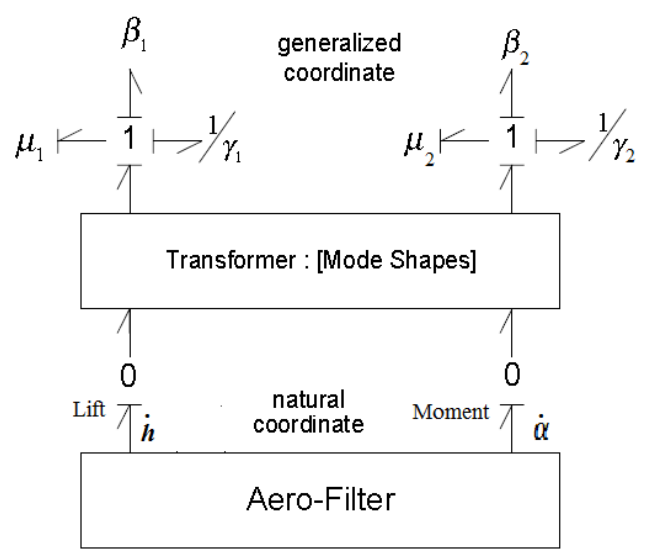

Fig. 9. Bond graph model of the aeroelastic system. The sources of effort represented by the Aero-Filter block as a function of the structural response, shows a flow-structure interaction.

It is advantageous to represent the equations of motion in modal coordinate system as the equations of motion can be represented in decoupled form. The two degree of freedom system in modal based coordinates will appear simpler in bond graph model as each mode can be considered as two sets of one-degree-of-freedom system as shown in Fig. 9. The external loads in the natural coordinate system are converted to modal forces through a sub-system of transformers containing modal matrix of mode shapes [4]. Through parallel/ zero junction arrangement and combined with the transformer subsystem, the natural coordinates can be transformed to generalised coordinate system and vice-versa. In this sense the inputs to the structure are the lift and the pitching moment acting on the natural coordinates of the aerofoil and the outputs of the structure are the dynamic response i.e. the velocities in transversal and rotational of the aerofoil.

The equivalent block diagram of the bond graph shown in Figure 9 for the structural part can be implemented using Simulink as shown in Figure 10.

\section{Simulation in Time Domain}

To start the simulation a small disturbance in the form of impulse i.e. a vertical force of $1^{-10} \mathrm{~N}$ with 0.1 second of duration is applied on the aerofoil. At the stability boundary where the wind speed is $15.5 \mathrm{~m} / \mathrm{s}$, the response of the structure is harmonic shown in Fig. 10. The disturbance in heaving direction excites not only the oscillation in heaving motion but in pitching as well. This phenomenon explains that there is an aeroelastic coupling between the two natural modes as perceived in Fig. 4.

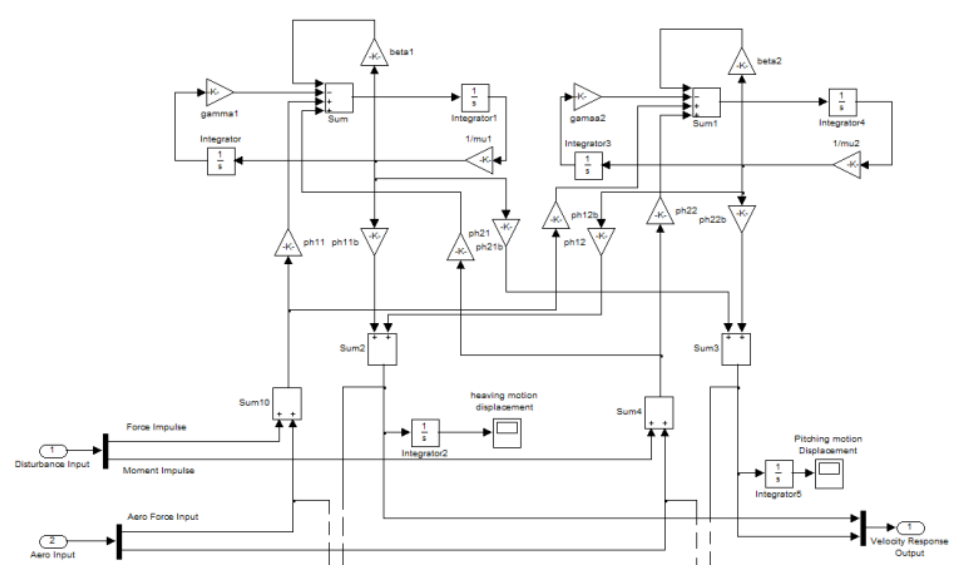

Fig. 10. Structural dynamics sub-system. This block diagram is the implementation of bond graphs in the platform of SimulinkMatlab.

Fig. 11 shows the flutter response that occurs at the wind speed slightly bigger than the critical speed. The oscillations grow exponentially divergent as the energy supplied from of the aerodynamic flow cannot be dissipated, and this vibration will lead eventually to the disintegration of the structure. The increase in energy of the oscillating aerofoil per cycle of flutter motion is expressed in terms of the work done by the self-excited forces. This increasing energy of vibration is intended to be converted to electrical energy through a power transducer. In bond graph model, the power can be calculated straight away as the product of the variable of effort (aerodynamic force) with the variable of flow (structure velocity).

In case of wind energy harvesting, the linear type of electric generator is mounted on the suspension system for the heaving mode of oscillation as explained in Figure 1. 


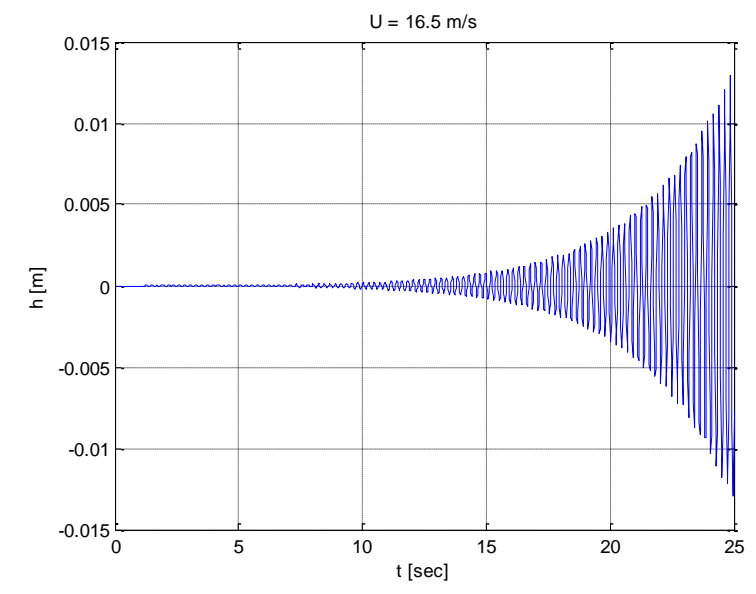

Figure 11. Divergent unstable flutter response in heaving motion at $U=16.5 \mathrm{~m} / \mathrm{s}$. This unstable self-excited vibration is triggered by a small impulse on the aerofoil in heaving direction. Both oscillations in heaving and pitching are generated.

The electrical load of the generator can be represented in additional mass, stiffness and damping of the system that will precede a higher critical speed of flutter boundary.

Increasing the wind speed above the flutter boundary will cause oscillations grow until a stable vibration where the nonlinear limit cycle is reached before the failure occurs. To avoid plastic deformation of the material of the support system and to be able to tap the wind energy in a stable condition a stopper mechanism to limit the motion distance of the fluttering aerofoil is applied. Theoretically the application of the stopper mechanism can be regarded as nonlinear stiffness of the aeroelastic system where the stiffness becomes infinitely high beyond a certain distance. The simulation in time domain using Simulink Matlab enables to apply this condition where the response values are restricted to certain amounts of range.

Figure 12 shows the fluttering aerofoil response in a limit cycle oscillation where the pitching motion is limited to the angle of 0.05 radians.

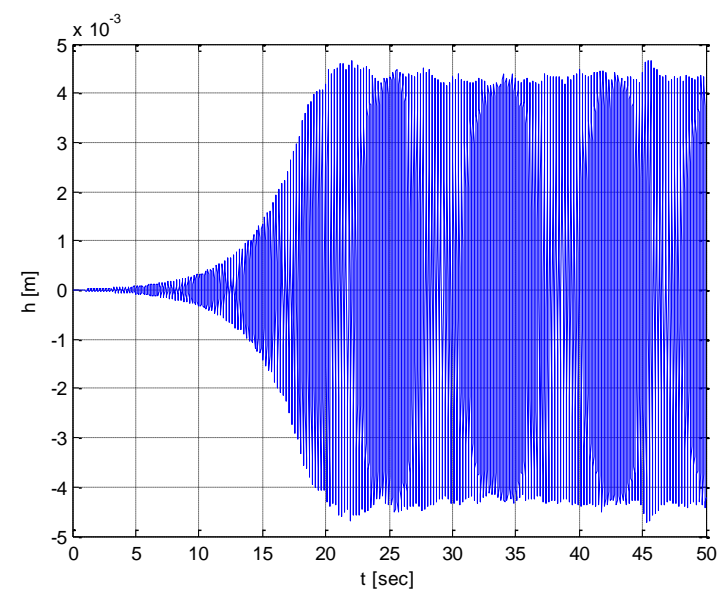

Figure 12. Limit cycle oscillation in heaving motion at $U=16.5$ $\mathrm{m} / \mathrm{s}$ with angle limitation in pitching motion of 0.05 radian.

\section{Conclusion}

The idea of the research is to propose a non-rotating turbine for harvesting wind energy by making use the aeroelastic behaviour of lifting surface structures where the flow energy is pumped out when the flutter occurs. In this paper the concept has been implemented into a realistic design and numerically analysed to quantify the extracted power.

The device is designed at a certain operating range of wind speed above flutter boundary and to have a stable oscillating motion where the wind energy is to be extracted, a stopper system is applied to limit the travelling distance in rotation.

The flutter boundary is the focus of the design with the strategy to find wind low speed of extent for an optimal working range of the device.

\section{Acknowledgement}

This research was financed previously under MEng Group Design \& Make (GDM). The first prototype was designed and built by a group of MEng students: Lu Kok Tiong, Yew Shu Hong, Lim Yeong Jian and Melissa C Chia. The project was afterwards continued through a short term research under Engineering Dean's UG Research Scheme during summer session 2012. The experiment set up and wind-tunnel test were conducted by the student of the research grant holder: Saad Javeed Aulia.

\section{References}

[1] F Fei, WJ Li, "A Fluttering-to-Electrical Energy Transduction System for Consumer Electronics Applications", Proceedings of the 2009 IEEE, International Conference on Robotics and Biomimetics, December 19-23, 2009, Guilin, China.

[2] YS Lee et al, "Suppressing Aeroelastic Instability Using Broadband Passive Targeted Energy Transfers, Part 2: Experiments", Journal of AIAA, 2007, DOI: $10.2514 / 1.28300$

[3] DC Karnopp, DL Margolis, RC Rosenberg, System Dynamics, Modeling and Simulation of Mechatronic Systems, 4th edition, John Willey \& Sons, Inc, 2005, Chapter 10, pp. 405.

[4] JR Right, JE Cooper, "Introduction into Aircraft Aeroelasticity and Loads", John Willey \& Sons, 2007, pp. 156-157.

[5] M Bryant, E Garcia, "Development of an Aeroelastic Vibration Power Harvester", Active and Passive Smart Structures and Integrated System 2009, Proc. of SPIE Vol 7288 728812-1.

[6] http://www.humdingerwind.com 